WellBeing International

WBI Studies Repository

$1-2013$

\title{
Social Learning in Juvenile Lemon Sharks, Negaprion brevirostris
}

\author{
Tristan L. Guttridge \\ University of Leeds \\ Sander van Dijk \\ University of Groningen \\ Eize Stamhuis \\ University of Groningen \\ Jens Krause \\ Leibniz-Institute of Freshwater Ecology and Inland Fisheries \\ Samuel Gruber \\ Rosenstiel School of Marine and Atmospheric Science
}

See next page for additional authors

Follow this and additional works at: https://www.wellbeingintlstudiesrepository.org/acwp_asie

Part of the Animal Studies Commons, Comparative Psychology Commons, and the Other Animal Sciences Commons

\section{Recommended Citation}

Guttridge, T. L., van Dijk, S., Stamhuis, E. J., Krause, J., Gruber, S. H., \& Brown, C. (2013). Social learning in juvenile lemon sharks, Negaprion brevirostris. Animal cognition, 16(1), 55-64.

This material is brought to you for free and open access by WellBeing International. It has been accepted for inclusion by an authorized administrator of the WBI Studies Repository. For more information, please contact wbisr-info@wellbeingintl.org.

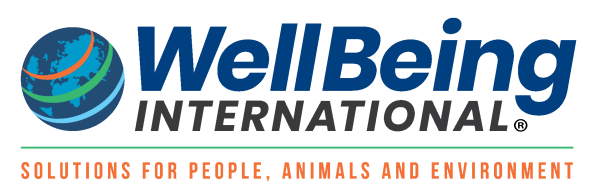


Authors

Tristan L. Guttridge, Sander van Dijk, Eize Stamhuis, Jens Krause, Samuel Gruber, and Culum Brown 


\title{
Social learning in juvenile lemon sharks, Negaprion brevirostris
}

\author{
Tristan L. Guttridge ${ }^{1,5}$, Sander van Dijk ${ }^{2}$, Eize J. Stamhuis ${ }^{2}$, Jens Krause ${ }^{3}$, Samuel H. Gruber ${ }^{4}$, \\ Culum Brown ${ }^{5}$ \\ ${ }^{1}$ University of Leeds \\ ${ }^{2}$ University of Groningen \\ ${ }^{3}$ Leibniz-Institute of Freshwater Ecology and Inland Fisheries \\ ${ }^{4}$ Rosenstiel School of Marine and Atmospheric Science \\ ${ }^{5}$ Macquarie University
}

\section{KEYWORDS}

local and stimulus enhancement, group living, social facilitation, social information use, Elasmobranchs

\begin{abstract}
Social learning is taxonomically widespread and can provide distinct behavioural advantages, such as in finding food or avoiding predators more efficiently. Although extensively studied in bony fishes, no such empirical evidence exists for cartilaginous fishes. Our aim in this study was to experimentally investigate the social learning capabilities of juvenile lemon sharks, Negaprion brevirostris. We designed a novel food task, where sharks were required to enter a start zone and subsequently make physical contact with a target in order to receive a food reward. Naive sharks were then able to interact with and observe (a) pretrained sharks, that is, 'demonstrators', or (b) sharks with no previous experience, that is, 'sham demonstrators'. On completion, observer sharks were then isolated and tested individually in a similar task. During the exposure phase observers paired with 'demonstrator' sharks performed a greater number of task-related behaviours and made significantly more transitions from the start zone to the target, than observers paired with 'sham demonstrators'. When tested in isolation, observers previously paired with 'demonstrator' sharks completed a greater number of trials and made contact with the target significantly more often than observers previously paired with 'sham demonstrators'. Such experience also tended to result in faster overall task performance. These results indicate that juvenile lemon sharks, like numerous other animals, are capable of using socially derived information to learn about novel features in their environment. The results likely have important implications for behavioural processes, ecotourism and fisheries.
\end{abstract}

\section{Introduction}

Group living among wild animals is a widespread phenomenon and can provide distinct behavioural advantages such as enhanced foraging, reduced predation risk and social learning (Krause and Ruxton 2002). In the context of animal research, social learning can be defined as any process through which one individual (the demonstrator) influences the behaviour of another individual (the observer) in a 
manner that increases the probability that the observer subsequently learns something (Hoppitt and Laland 2008). It is often assumed that social learning is more common and restricted to 'intelligent' or 'large-brained' taxa (Brown and Laland 2011). Social learning is, however, widespread and has been broadly documented in a variety of taxonomic groups such as mammals, birds, fishes and insects (Leadbeater and Chittka 2007; Hoppitt and Laland 2008; Brown and Laland 2003).

There is now extensive experimental evidence that social learning plays an important role in the development of behaviour in many fishes and frequently underlies differences in the behavioural repertoires of different populations (Brown and Laland 2001; Laland et al. 2011). The mechanisms that result in social learning can vary widely. For example, social learning through 'stimulus enhancement' occurs when the behaviour of an individual attracts the attention of another to a particular stimulus, about which the naïve individual subsequently learns something. 'Social facilitation' occurs when the behaviour of one individual induces an identical behaviour in another individual and the latter then learns something via the expression of this behaviour (Brown and Laland 2011). 'Guided learning' refers to instances in which, by following a knowledgeable animal, an individual is exposed to similar features of the environment and comes to learn the same behaviour. 'Observational conditioning' occurs when the response of a demonstrator to a stimulus elicits a matching response on the part of an observer, who simultaneously perceives the original stimulus and effectively learns that the response is appropriate. For instance, rhesus monkeys (Macaca mulatta) can acquire a fear of snakes when they witness experienced monkeys behaving fearfully in the presence of a snake (Mineka and Cook 1988). A variety of other processes, some assuming more sophisticated psychological underpinnings, can also facilitate social learning in animals, described variously as imitation, goal emulation, copying, etc. (see Heyes 1994; Hoppitt and Laland 2008, for reviews). In all cases, social learning is said to occur only if the naïve animal maintains the learnt behaviour in the absence of the demonstrator. Understandably, most research on fish social learning has tended to focus on small freshwater Teleost species, such as guppies (Poecilia reticulata) and sticklebacks (Gasterosteidae), that are easy to manipulate and maintain in laboratories (Laland et al. 2011). These studies have identified social learning across a variety of behavioural contexts, from finding food and mates, to avoiding predators and navigating through space (see Brown and Laland 2011; Laland et al. 2011 for reviews). However, despite extensive research, there seem to be few experimental studies of social learning where researchers can isolate exactly which type of social learning process is operating (Hoppitt and Laland 2008).

For marine fishes, social learning research has tended to focus on commercially important species, such as salmon (Salmonidae), pollock (Theragra chalcogramma) and cod (Gadus morhua) (Ryer and Olla 1992; Vilhunen 2006; Brown and Laland 2011). This is mainly because of the interest in aquaculture and the need to devise rearing methods to enhance survival of captive-bred stocks after release into the wild (Brown and Laland 2001; Brown and Day 2002). There are no such experiments investigating the social learning tendencies of large marine predators, such as sharks. The most recent reviews on the topic discuss no research from any cartilaginous fishes (Brown and Laland 2011).

Sharks form aggregations, and it is apparent that the behaviour and organisation of these can vary greatly between species (Springer 1967; Jacoby et al. 2011). Sharks may form polarised schools (Sphyrna lewini; Klimley \& Nelson 1981), loosely milling groups with no observable coordination (Carcharhinus amblyrnchos; Economakis \& Lobel 1998) or resting groups (Heterodontus portusjacksoni; Powter \& Gladstone 2009). Group formation is thought to provide behavioural advantages (see Jacoby et al. 2011 for a review). Some researchers have also hypothesised that sharks are capable of social learning (Klimley and Nelson 1981; Bres 1993; Jacoby et al. 2011) and that groups can form through active social preference, not just through an attraction to a mutual resource (Guttridge et al. 2009a; Jacoby et al. 2010). Shark 'feeding frenzies' are often referred to as an example of social facilitation 
(Nelson and Johnson 1980). As shark density increases around prey, competition also increases leading to more active swimming and enhanced, excitatory behaviours (particularly from new arrivals) via cyclical social reinforcement. Robbins et al. (2011) emphasised the importance of considering shark social behaviours and densities when designing and implementing shark deterrents.

Although social learning is an important function of group living in many animal taxa (Heyes 1994), no study has empirically tested whether sharks, or any cartilaginous fish, use socially acquired information (Guttridge et al. 2009b). This is surprising considering the importance of determining the evolutionary origins of social learning in vertebrate taxa and potential conservation and management implications, such as understanding the function of aggregations (Jacoby et al. 2011).

The lemon shark (Negaprion brevirostris) is a large-bodied coastal species that occurs in the Eastern Pacific and Western and Eastern Atlantic (Compagno 1984). At our study site (Bimini, Bahamas), juveniles $(<1 \mathrm{~m})$ are abundant and have been successfully used in captive experimental studies (Gruber and Schneiderman 1975; Gruber 1982; Guttridge et al. 2009a). Lemon sharks form aggregations at various life stages (Wetherbee et al. 2007; Kessel 2010; Guttridge et al. 2011) and have previously been trained successfully to classical and operant conditioning regimes in captivity (Clark 1959; Gruber and Schneiderman 1975). These characteristics make the juvenile lemon shark an excellent model species to experimentally investigate social learning processes in cartilaginous fish.

In this study, we designed a novel food task and trained demonstrator sharks to solve it. Observer sharks were allowed to interact with and observe either (1) pre-trained sharks (i.e. 'demonstrators') or (2) sharks with no previous experience (i.e. 'sham demonstrators'). During this exposure phase, task-related and social behaviours of observer sharks were recorded and compared between the two groups. On completion, observer sharks were then tested in isolation to determine whether they had retained any of the task-specific behaviours. We predicted that during the testing phase the observer sharks that had been exposed to knowledgeable demonstrators would complete the task more often than observer sharks exposed to sham demonstrators. More specifically during the exposure phase, we expected observers trained with demonstrators to visit the target and target zones more often, make more transitions between the start zone and the target, and perform fewer non-task-related behaviours such as leading and following. Feeding sharks show enhanced activity and little, if any, social organisation (Nelson and Johnson 1980; Jacoby et al. 2011); thus, observers should be focussed on monitoring conspecific taskrelated behaviours and enact fewer social behaviours. Ultimately, we expected observers trained with demonstrators to complete the task more regularly during the individual test phase (after the removal of demonstrators) than those trained with sham demonstrators.

\section{Materials and methods}

Study site and study sharks

The study was conducted at Bimini, Bahamas $\left(25^{\circ} 44^{\prime} \mathrm{N}, 79^{\circ} 16^{\prime} \mathrm{W}\right)$, a small series of islands approximately $85 \mathrm{~km}$ east of Miami, Florida, USA. A total of 18 juvenile lemon sharks (mean pre-caudal length, PCL $=$ $55 \pm 8 \mathrm{~cm}$ ) were used in our experiments. Sharks were captured using gillnets and transported immediately to a large holding pen. On arrival, each shark was restrained in a trough $(10 \times 100 \mathrm{~cm})$ to allow tag attachment and pre-caudal length (PCL) measurement (see Dibattista et al. 2008). Each shark was marked with a unique colour-coded T-bar tag to provide external individual identification (Floy Tag Manufacturing) through the first dorsal fin. All sharks used were housed outdoors in circular $(10 \mathrm{~m}$ diameter) holding pens built just offshore in shallow $(0.5-1.5 \mathrm{~m})$ sand-bottom flats (see Dibattista et al. 2008). The pens were built of plastic mesh $(5 \times 5$-cm diamond-shaped holes), which exposed the sharks to natural ambient conditions (e.g. tidal and lunar cycles). All sharks were given 1 week to acclimatize to 
captive conditions and were fed to satiation during non-experimental periods on a mixed diet of fresh and frozen local fish (Sphyraena barracuda). Sharks were handled using dip nets and transported between pens in plastic tubs (100 I). No sharks died during the experiments, and all were released at their site of capture. A permit (no: MAF/LIA/22) to conduct scientific marine animal research was supplied by the Department of Marine Resources, Bahamas.
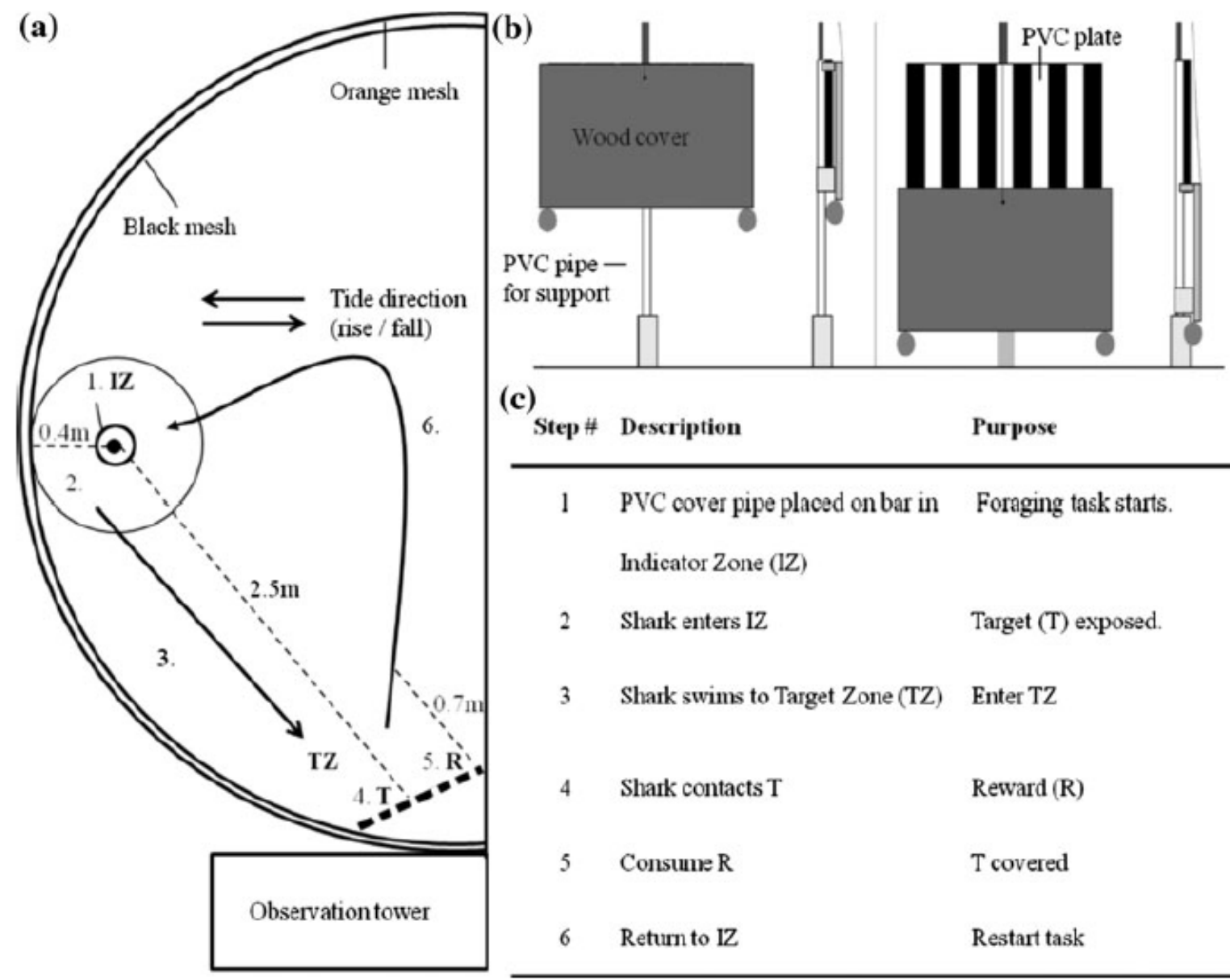

(c) Step \# Description Purpose

1 PVC cover pipe placed on bar in Foraging task starts. Indicator Zone (1Z)

2 Shark enters IZ Target (T) exposed.

3 Shark swims to Target Zone (TZ) Enter TZ

4 Shark contacts T Reward (R)

5 Consume $\mathrm{R} \quad$ T covered

6 Return to IZ Restart task

Fig. 1 Set-up of social learning experiment: a positions and measurements of zones, target and reward; b schematic of the target mechanism showing covered and exposed position as well as side and front views; and $c$ steps of the food task or trial

\section{Experimental set-up and sharks}

All sharks were assigned to one of two age groups based on length (group 1, $n=11$ : standard length = 45-55 cm, age 0-1 years; group 2, $n=7$ : standard length $=60-70 \mathrm{~cm}$, age 2-3 years). Within these groups, we constructed experimental quartets consisting of size-matched observer-demonstrator (O-D) pairs and observer-sham demonstrator (SO-SD) pairs. Two sharks were reused as Ds, and a total of five experimental quartets were tested. Two identical experimental pens like the holding pens described 
above (diameter 5 m, Fig. 1) were constructed and placed $20 \mathrm{~m}$ apart. Fine black meshing $(2 \times 2-\mathrm{mm}$ square-shaped holes) was secured internally to prevent other organisms from entering. Pens were oriented so that tidal flow moved horizontally across the experimental area minimising the spread of olfactory cues throughout the pen (Fig. 1).

Each pen was equipped with a manually operated PVC target and indicator, both black and white banded (Fig. 1). Target and indicator zones were demarcated by sand-filled, green hose pipe. These zones were used to assess the use of areas relevant to the task (i.e. visits). Food rewards were tethered to a PVC feeding pole. An observation tower (height $2 \mathrm{~m}$ ) was positioned outside the pen allowing for control of the target, for reward and to facilitate recording of shark behaviour during trials (Fig. 1).

During experimental periods, individual shark total daily food intake was equivalent to $2 \%$ of their body weight. This percentage is known to sustain captive sharks and ensured that motivation to feed remained consistent between experiments (Gruber 1982). Sharks from group one were fed $\sim 25 \mathrm{~g}$ per day and group two $\sim 60 \mathrm{~g}$ per day. All sharks were fed pre-cut and weighed frozen white muscle fish pieces. All experiments were completed during daylight hours ( 9 a.m. -4 p.m.) over the low tide $( \pm 3 h)$ and in light wind conditions $(<15 \mathrm{~km} / \mathrm{h})$. The experimental protocol is described below and listed in Table 1 and Fig. 1.

\section{Training of demonstrator sharks}

Our first two $\mathrm{D}$ sharks were moved from the holding pen to one of the experimental pens, given $24 \mathrm{~h}$ to acclimatise and then trained to complete the following steps: enter indicator zone after placement of indicator cover (moving into this zone exposes the target), move to the target zone and make contact with the target. Upon contact with the target, a food reward was provided in front of the target. Once the food was eaten, the apparatus was reset and a new trial began (Fig. 1). The indicator cover was only placed at the start of the first trial to signal to the shark that a trial had begun. It was removed after all trials were completed, thereby signaling the end of training. Throughout this initial training, extra fish pieces and olfactory cues (fish concentrate) were used to enhance feeding motivation, reducing the time taken to train sharks to associate the target with food. A shark was considered sufficiently trained to act as a ' $D$ ' when the time between entering the indicator zone and contacting the target was completed in $<15 \mathrm{~s}$, on ten consecutive trials. Two $O$ sharks that took part in the experiment were subsequently trained to the above criteria and then reused as D sharks, one on two occasions. A total of four D sharks were used with one individual used as a D shark twice.

Table 1 Description of experimental steps

\begin{tabular}{|c|c|}
\hline Task & Description \\
\hline Shark capture & All sharks captured, processed and housed in holding pens \\
\hline D training & $\begin{array}{l}\text { D shark moved to individual experimental pen, training started and continued until } \\
\text { criterion reached, } 10 \text { consecutive trials in }<15 \mathrm{~s} \text { from } \mathrm{IZ} \text { to contact target }\end{array}$ \\
\hline O and SO shark exposure & $\begin{array}{l}\text { O-D and SO-SD sharks moved to experimental pens and exposure to D and SD } \\
\text { sharks begins. Twenty trials across four sessions ( } 10 \text { trials per day over } 2 \text { days). O or } \\
\text { SO sharks were required to contact the target in< } 30 \mathrm{~s} \text { in order to receive a reward. } \\
\text { Only D sharks received a food reward }\end{array}$ \\
\hline $\mathrm{O}$ and SO shark testing & $\begin{array}{l}\text { D and SD sharks removed. O and SO sharks commenced solitary testing. Thirty trials } \\
\text { across six sessions ( } 10 \text { trials per day over } 3 \text { days). O and SO sharks continued } \\
\text { training until criterion was met, as above and then used as the next two D sharks }\end{array}$ \\
\hline Replicate & $\mathrm{O}$ and SO training/testing conducted (as above) with new D and SDs \\
\hline Release & On completion of experiments all sharks fed to satiation and released \\
\hline \multicolumn{2}{|c|}{$\begin{array}{l}\text { All sharks given } 24 \mathrm{~h} \text { to acclimatise to pen after disturbance, that is, movement from one pen to another or removal o } \\
\text { conspecific }\end{array}$} \\
\hline
\end{tabular}


During this phase, $O$ and SO sharks were exposed to $D$ and SD, respectively. Shark quartets (O-D and SO-SD pairs) were moved from holding to experimental pens (one pair per pen), where they were given $24 \mathrm{~h}$ to acclimatise. $\mathrm{O}$ and SO sharks then interacted with and observed their D or SD partner shark completing four sessions of five trials each split over two days, for a total of 20 trials. D and SD sharks started trials by entering the indicator zone (after indicator cover placement), which exposed the target. On entering the indicator zone, D or SDs were given $30 \mathrm{~s}$ to make contact with the target in order to receive a food reward. This $30 \mathrm{~s}$ period was chosen to increase the probability of the $D$ sharks demonstrating the task, as they were previously trained to complete it in $\backslash 15 \mathrm{~s}$. If no contact was made, no reward was given, the target was re-concealed, and D or SD sharks were then required to re-enter the indicator to expose the target again. At no point did SD sharks contact the target within this time period, so there was no opportunity for the SO sharks to learn socially that approaching the target delivered food. Each session continued until D or SD sharks successfully entered the indicator zone on five occasions, or a session ceiling time of $1 \mathrm{~h}$ was reached. O or SO sharks did not receive food rewards during these trials. After completion, D and SD sharks were transferred to holding pens and $O$ and SO sharks were retained for individual testing.

\section{Testing phase}

After a 24-h solitary acclimation period, $\mathrm{O}$ and SO sharks were tested individually for retention of the task. Sharks were tested over 10 trials per day for 3 days across six sessions for a total of 30 trials. The protocol for this testing followed the steps described in Fig. 1 with one change that reduced the task difficulty: When $\mathrm{O}$ or SO sharks entered the indicator zone and the target was exposed, a food reward was immediately placed in front of it. The sharks were not required to contact the target in order to receive a food reward. Once the reward was consumed, the target was re-concealed (trial end time) and the shark was required to re-enter the indicator zone in order to start the second trial. Each session continued until five trials were initiated or a session ceiling time of $1 \mathrm{~h}$ was reached. The decision to reduce the difficulty of the task was made due to the lengthy amount of time taken to train our initial $D$ sharks.

\section{Video analysis}

All trials were recorded using a digital video camera (Panasonic TZ1) and the footage converted and imported to video analysis software 'The observer XT' (Noldus 2007). An ethogram of social and taskrelated behaviours was constructed based on observations made during this experiment and common behaviours, such as follow formations, observed in previous studies (Myrberg and Gruber 1974; Guttridge et al. 2011; see Table 2). These behaviours were then recorded through 'The Observer XT', enabling the calculation of frequencies and durations for various task-related and behavioural measures (see Table 3).

\section{Data analysis}

In order to assess the task performance of sharks during both exposure and testing phases, we calculated session (exposure and test phases) and trial (test phase) durations. For our exposure phase, session duration was calculated as the latency from initial indicator zone entrance (i.e. first time the $D$ or SD shark entered the indicator zone) to trial five target contact (i.e. the fifth time the D or SD shark made contact with the target), or when five trials had been triggered (i.e. D or SD shark entered the indicator zone on five occasions, even if they failed to make contact with the target within the 30-s time period), or a ceiling time of $1 \mathrm{~h}$ was reached. Using these three criteria allowed us to include sharks in our analysis that did not make contact with the target or enter the indicator zone, which were behaviours that were an 
indication of poor performance. For our testing phase, $\mathrm{O}$ and SO sharks were not required to contact the target in order to receive a food reward. Therefore, the session duration was calculated as the latency from initial indicator zone entrance to reward consumption, or when five trials were triggered or a ceiling time of $1 \mathrm{~h}$ was reached. For this phase, we also calculated individual trial durations using the latency from indicator zone entrance to reward consumption (i.e. individual trial completion). Note in our testing phase, not all $\mathrm{O}$ or SO sharks entered the indicator zone to initiate trials. Therefore, analyses comparing the time taken from indicator zone to target zone or reward consumption between $\mathrm{O}$ and SO sharks only included sharks that initiated the start of a trial. Furthermore, we determined whether the number of transitions made between the indicator and target zone within specific time frames (e.g. 5 and $15 \mathrm{~s}$ ) differed between Os paired with D or SD sharks. A duration of $15 \mathrm{~s}$ was selected because this was the time within which the $D$ sharks were expected to move from the indicator zone to the target zone based on the learning criterion previously set and $5 \mathrm{~s}$, because sharks would need to swim quicker than normal cruising speeds ( 0.25 body lengths per $s$ ) in order to get from the indicator zone to the target zone. Nonparametric analyses were used throughout due to low replicates and non-normal data distributions. All statistical analyses were carried out in $\mathrm{R}$, version 2.12.1 (freeware available at www.r-project.org).

Table 2 Ethogram of juvenile lemon shark experimental behaviours

\begin{tabular}{|ll|}
\hline Behaviour & Definition of behaviour \\
\hline Following & $\begin{array}{l}\text { Sharks were within } 2 \text { body lengths of each other. Trailing sharks mimic the directional } \\
\text { changes of the lead individual. Both lead and trailing individuals were recorded }\end{array}$ \\
Accelerated swimming & $\begin{array}{l}\text { Shark swimming faster than 0.5 body lengths/s (Nixon and Gruber 1988; Bushnell } \\
\text { et al. 1989) }\end{array}$ \\
Indicator zone entry & Shark enters the indicator zone with its entire head (tip of snout to gill slits) \\
Target zone entry & Shark enters the target zone with its entire head (tip of snout to gill slits) \\
Contact target & Shark makes physical contact with the target using snout tip \\
Contact cover & Shark makes physical contact with the target cover using snout tip \\
\hline
\end{tabular}

Table 3 Ethogram of behaviours measured

\begin{tabular}{|ll|}
\hline & Measure \\
\hline Following or leading & \% of trial time \\
Accelerated swimming & Trial time \\
Indicator and target zone entry & Number of visits \\
Contact target and cover & Number of total contacts and number per minute across all trials \\
Indicator to target contact & Duration (s) \\
Indicator to target zone & Number of transitions within 5 and $15 \mathrm{~s}$ time frames and duration (s) \\
Session and trial completion & Duration (s) \\
\hline
\end{tabular}

\section{Results}

Exposure phase

During exposure trials, D sharks entered the indicator zone to initiate trials in 97/100 individual task trials. Ds then moved from the indicator zone and contacted the target in $<30 \mathrm{~s}$ in 94/97 trials. The performances of Ds were also consistent as we found no difference in the time to contact the target from the indicator zone across all sessions (Kruskal-Wallis rank-sum test, $X^{2}=3.2, d f=3, P=0.362$ ). As 
expected, D sharks completed food task sessions significantly faster than SD sharks (Wilcoxon signedranks test, $P<0.01$, Fig. 2). Interestingly, although SD sharks entered the IZ on 90/100 trials, thereby triggering exposure of the target, they never contacted the target and so no food reward was delivered.

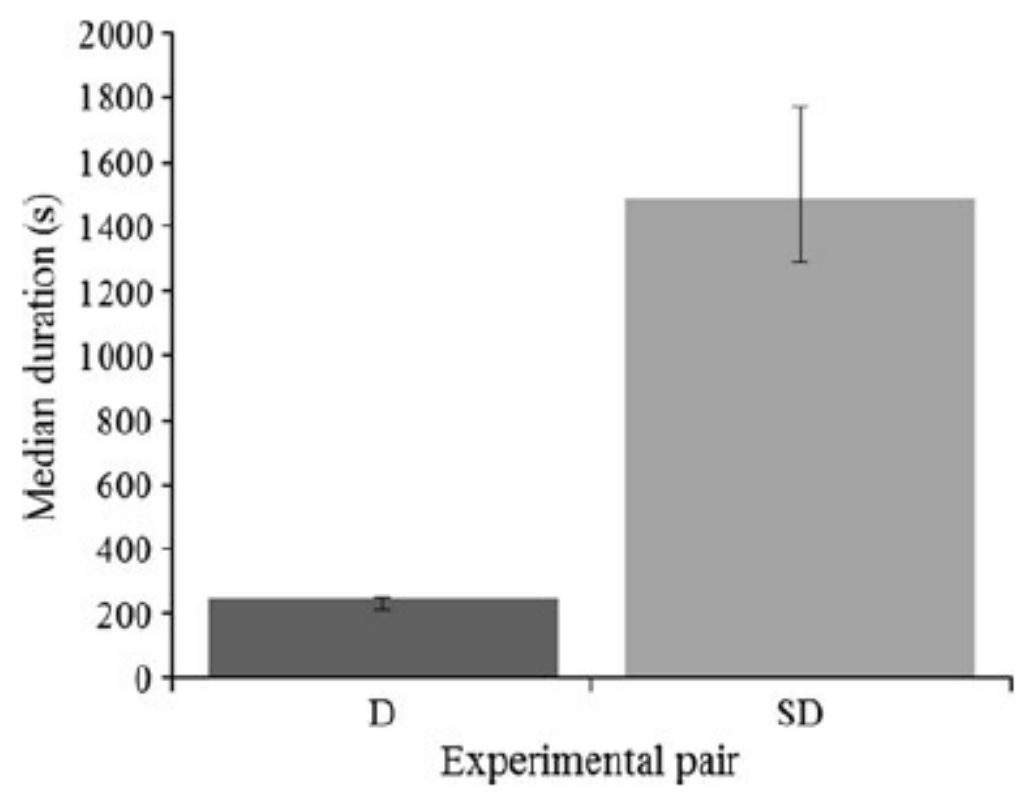

Fig. 2 Exposure phase: median ( \pm interquartile range) duration to complete five trials (one session), of the novel food task, by two juvenile lemon shark pairs (observer-demonstrator and sham observer-sham demonstrator). $P<0.01$, Wilcoxon signed-ranks test, $n=5$

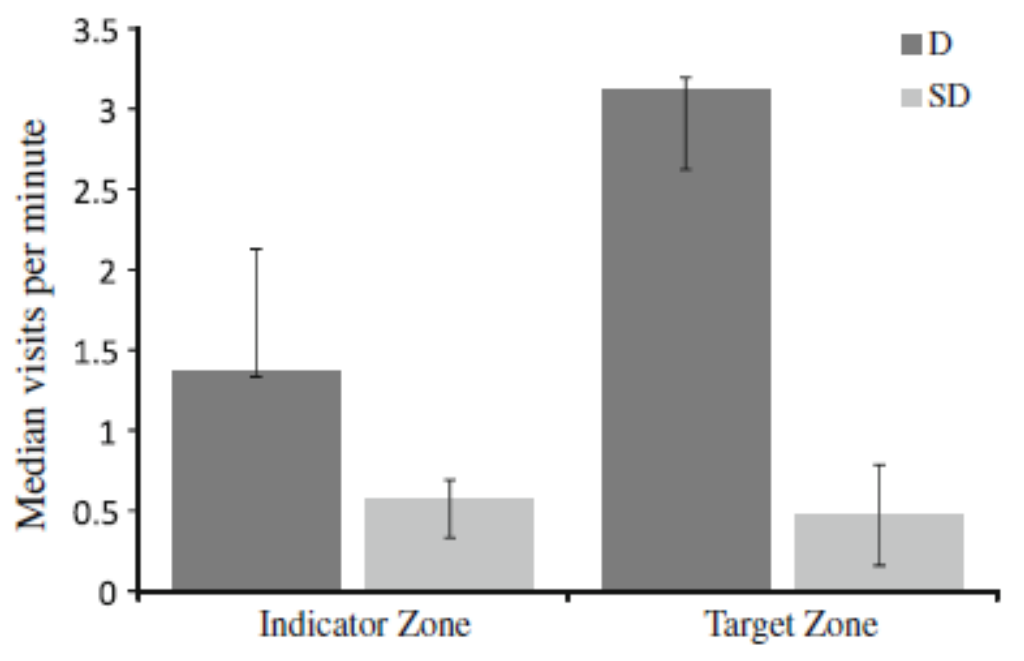

Fig. 3 Exposure phase: median ( \pm interquartile range) number of visits made per minute to the indicator zone (IZ) and the target zone (TZ) by observer juvenile lemon sharks paired with demonstrators (D) or sham demonstrators (SD). PI0.01 in both cases, Wilcoxon signed-ranks test, $n=5$ 
O sharks visited the indicator and target zones on significantly more occasions than SO sharks (Wilcoxon signed-ranks test, IZ: $W=25, P<0.01$; TZ: $W=25, P<0.01$, Fig. 3). The number of transitions (movement of shark from indicator zone to target zone within 5-s and 15-s time frames) per minute was also more frequent for $O$ compared with SO sharks (Wilcoxon rank-sum test, $5 \mathrm{~s}: W=25, P<10.05 ; 15 \mathrm{~s}$ : $W=25, P<0.01$ ). See supplementary videos 1 and 2 , respectively, for examples of $O$ and SO exposure trials (exposure trial day 1 , trial 7 ).

O sharks also made more physical contacts with the target (number of contacts $=25$, mean number of contacts/trial \pm standard deviation $=2.3 \pm 1.8$ ) and the target cover (number of contacts $=50$, mean number of contacts/ trial \pm standard deviation $=3.1 \pm 2.3 \mathrm{SD}$ ) during trials than SO sharks, which made no such contacts. Furthermore, SO sharks spent significantly more time (median \% of trial duration) during trials following and leading in social pairs than $O$ sharks (Wilcoxon signed-ranks test, Leading: $W=$ 2, $P<0.05$; Following: $W=0, P<0.01$ ). However, we did not detect a difference between the time spent in lead or trail positions for O-D and SO-SD pairs (Wilcoxon signed-ranks test, O-D: $W=5, P=0.134$; SO-SD: $W=11, P=0.84$ ). Finally, $O$ sharks were also recorded swimming at accelerated speeds (median trial duration spent swimming accelerated $=4 \mathrm{~s}$, upper and lower quartile $=33.75$ and 0 ), whereas only one such event (duration $=2 \mathrm{~s}$ ) was documented for SO sharks.

\section{Testing phase}

O sharks completed 140/150 individual food task trials, across six sessions with four of five individuals completing all trials, whereas SO sharks completed 110/150 individual trials, across six sessions with only one shark completing all trials. O sharks also showed a general trend to complete the task faster than SO sharks, for duration of trial 1 (Wilcoxon rank-sum test :Trial $1 W=20, P=0.143$ ), session 1 (Wilcoxon rank-sum test: Session $1, W=3, P=0.059$ ) and all sessions combined (O: median time per session $=9.3$ min, upper and lower quartile $=11.9$ and 7; SO: median time per session $=26$ min, upper and lower quartile $=55.4$ and 25.6) (Wilcoxon rank-sum test, $W=21, P=0.09$ ).

Both $\mathrm{O}$ and SO sharks moved from the indicator zone to the target zone in trial five of each session significantly faster than in trial one (Wilcoxon rank-sum test, O: $W=21, P<0.05$; SO: $W=21, P<0.05$ ), suggesting that both treatment groups improved their performance through experiential rather than social learning, within sessions. However, O sharks were not faster than SO sharks in moving from the indicator zone to the target (Wilcoxon rank-sum test, $W=29, P=0.09$ ). When combining trials across all sessions, SO sharks showed a significant improvement in the time to travel from the indicator zone to the target zone (Kendall' rank correlation, $\tau=-0.30, P=0.019$, Fig. 4), whereas no such improvement was detected for $O$ sharks (Kendall' rank correlation, $\tau=-0.20, P=0.12$, Fig. 4). This is largely due to the fact that SO first trial was consistently longer than $100 \mathrm{~s}$, whereas $\mathrm{O}$ sharks consistently completed the task in less than $100 \mathrm{~s}$ during their first trial. We also found that $\mathrm{O}$ sharks made significantly more physical contacts with the target and target cover per minute across all trials than SO sharks (Wilcoxon rank-sum test, $W=$ 23, $P<0.05$, Fig. 5).

\section{Discussion}

We found some evidence of social learning in juvenile lemon sharks in a novel food context. During exposure to demonstrators (Ds), observer (O) sharks paired with Ds performed a greater number of taskrelated behaviours, such as visiting the indicator and target zones and making physical contact with the target and the target cover, than sham observers (SO) paired with sham demonstrators (SDs). When tested in isolation, $\mathrm{O}$ sharks completed a greater number of trials and made contact with the target significantly more often than SOs. Such experience also tended to result in faster overall task performance; however, this was not statistically significant. Nevertheless, our results do indicate that 
juvenile lemon sharks, like numerous other animals (Heyes 1994; Leadbeater and Chittka 2007; Hoppitt and Laland 2008), are capable of using social information that likely has important implications for behavioural processes, such as finding food.
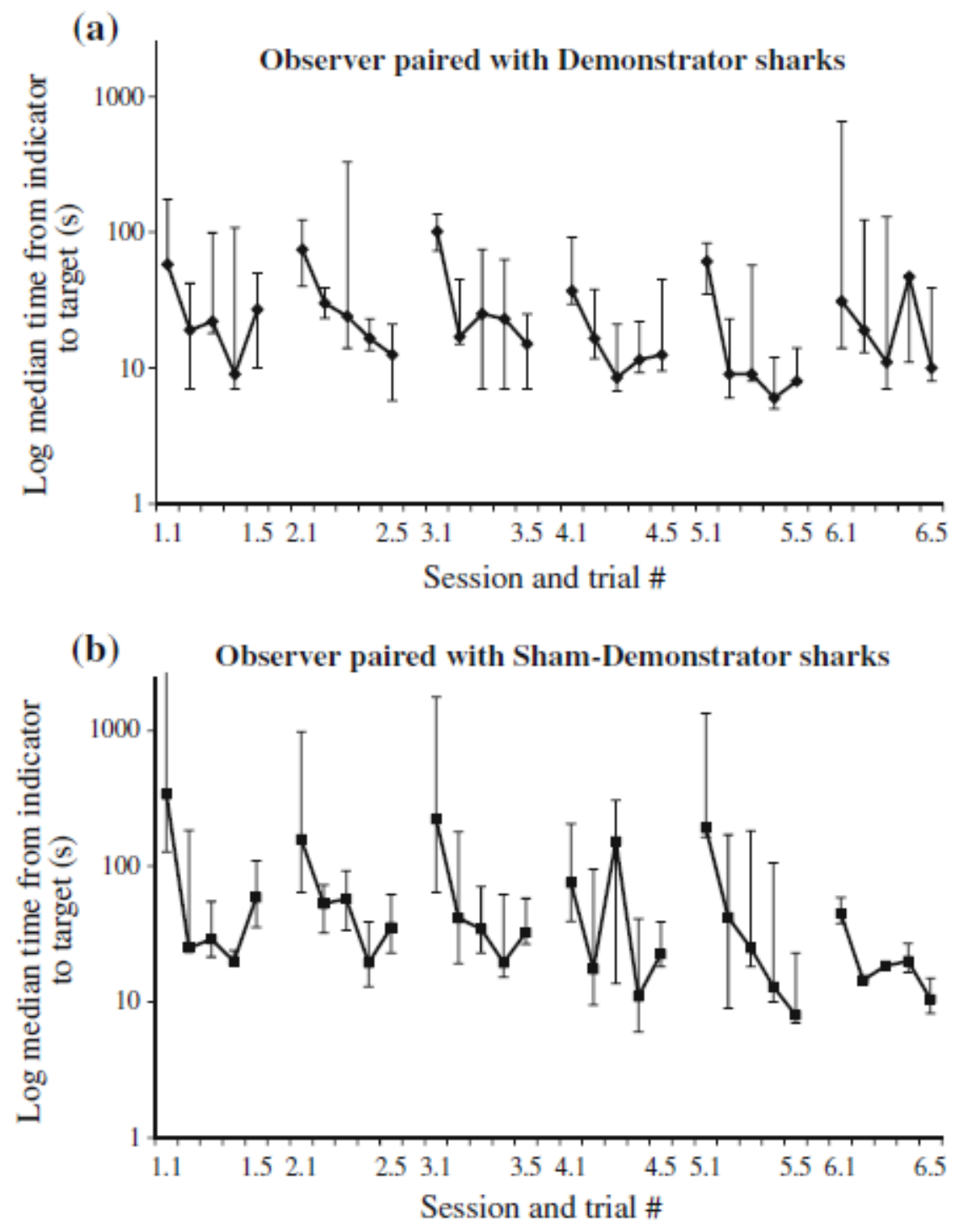

Fig. 4 Testing phase: log median ( \pm interquartile range) time per trial from the indicator zone to the target zone by observer sharks previously paired with a demonstrators (D) or b sham demonstrators (SD)

During our exposure phase, $\mathrm{O}$ sharks made significantly more transitions between the indicator zone and target zone (within 5-s and 15-s time frames per minute) than SO sharks. This result is consistent with the number of visits made to each of these zones, which was significantly greater for $O$ than SO sharks. It is possible that the increased frequency of visits made to the target zone was a consequence of sharks being attracted to the olfactory cues released by the food reward, leading to sharks learning individually. However, we believe such influences would be minimal due to the semi-natural nature of our pens and 
their orientation across the tidal flow, resulting in any olfactory cues being rapidly removed from the area. In addition, the fact that $O$ sharks also visited the indicator zone, more often than SO sharks suggests that they were attracted to it by the excited behaviour of their Ds. Moreover, the transition from indicator zone to target zone and subsequent contact with the target indicates that $O$ sharks trained with Ds were beginning to learn the contingencies between the zones and the food reward, essentially completing the task. The supplementary video of O-D sharks shows this clearly even though this was only trial 7 of the 20 exposure trials. There is an abundance of evidence, from both laboratory and wild studies, showing that Teleost fishes can learn predator escape routes and foraging locations and routes from conspecifics (Brown and Laland 2011; Laland et al. 2011). These and previous studies on coral reef fishes (Helfman and Schultz 1984) illustrate that some fish behaviours, both in the laboratory and wild, are underpinned by social learning processes (Laland et al. 2011). Sharks exhibit a variety of movement strategies, with some species migrating large distances and others displaying site fidelity (Bonfil et al. 2005; Speed et al. 2010). The extent to which these strategies are influenced by the use of social information remains completely unexplored and will be an exciting avenue for future research.

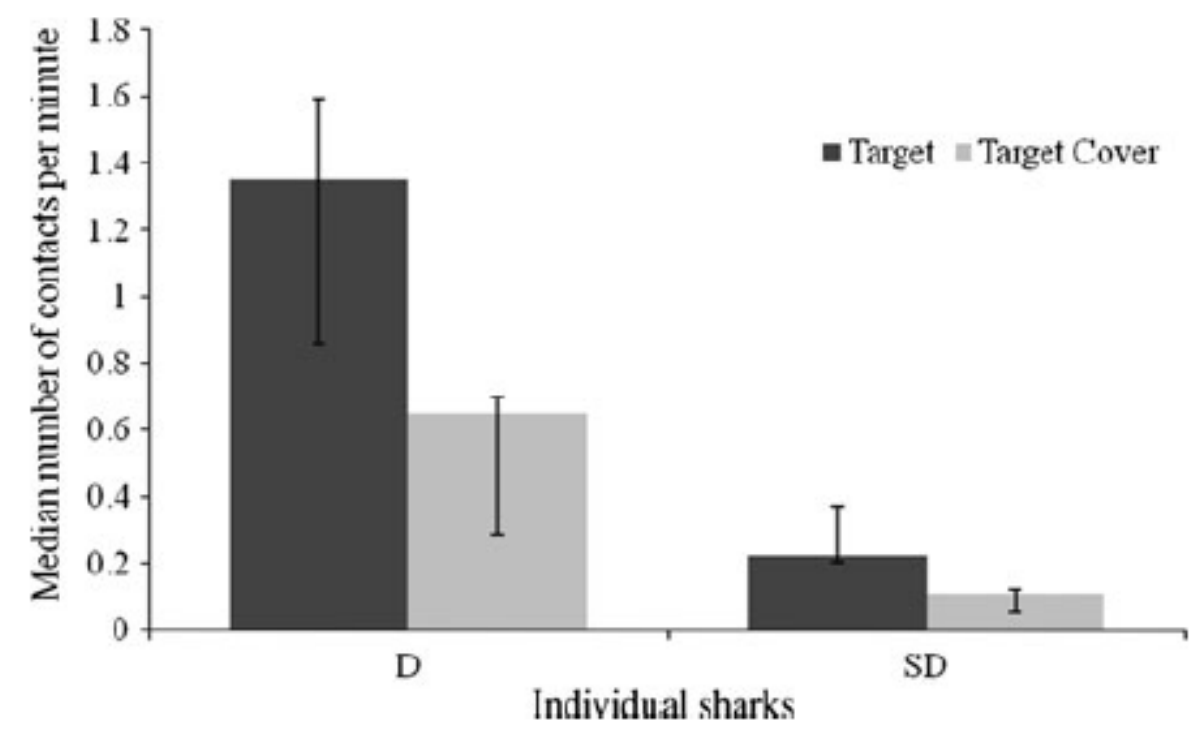

Fig. 5 Testing phase: median ( \pm interquartile range) number of physical contacts made with the target and the target cover by individual observer sharks previously paired with demonstrators (D) or sham demonstrators (SD). $P<0.05$ in both cases, Wilcoxon signed-ranks test, $n=5$

In accordance with our predictions, $O$ sharks paired with SDs spent more time performing social behaviours, such as following, than $O$ sharks paired with Ds. Such behaviours are commonly observed in both wild and captive juvenile lemon sharks (Guttridge et al. 2009a, 2011). Although O sharks paired with D sharks were observed in such follow formations, the low frequency of them indicates to us that these sharks were more focussed on task-related behaviours, such as moving into the indicator zone and target zone or making contact with the target, than schooling. Interestingly, O sharks were also observed to swim in an accelerated manner during the training phase, matching their Ds excited behaviour, whereas such accelerated behaviours were only observed on one occasion for SO sharks paired with SDs. Again, it is also possible that such behavioural changes were due to the $O$ sharks detecting olfactory cues emitted from the food reward; however, due to the reasons mentioned above, we do not believe that this was generally the case. Future experiments should be designed to avoid the use of food cues, with 
stricter protocols in order to completely rule out their influence on behaviour. For other animals, monitoring and reacting to the behaviours of conspecifics has been extensively documented, in the context of foraging and anti-predatory behaviour (see Hoppitt and Laland 2008; Brown and Laland 2011 for reviews). Our results imply that juvenile lemon sharks are sensitive and attracted to the excitatory and foraging behaviours of conspecifics.

Results from our testing phase showed that $O$ sharks made significantly more physical contacts with the target and target cover per minute across all trials, compared with SO sharks. This result is in accordance with our exposure phase, indicating that $O$ sharks learnt that the novel stimulus or target was in this context biologically relevant. This suggests that the learning of instrumental conditioning tasks will likely improve through exposure to trained individuals. For Teleost fishes, such techniques have been used successfully to control food intake and promote foraging behaviour of naïve/new individuals (Brown and Laland 2011; Fernö et al. 2011) and could be adapted to promote feeding of sharks in captivity.

Results from our testing phase also showed that $O$ sharks completed more individual trials across all sessions than SO sharks. Although there was a general trend for $O$ sharks to complete trials within sessions and overall session durations faster than SO sharks, such differences were not statistically significant. $\mathrm{O}$ and $\mathrm{SO}$ sharks also improved equally within sessions, with both moving significantly faster from the indicator zone to target zone in trial five than they did in trial one. However, despite these withinsession improvements, indicative of experiential learning, it was only SO sharks that showed significant improvement across all sessions, no such improvement was detected for $O$ sharks. This occurred because the $O$ sharks were already demonstrating a higher performance during the first session (the only session in which social information had pronounced influence) than the SO sharks and thus show less improvement as the trials progressed. Regrettably, our decision during the testing phase to place the food in front of the target, immediately after being revealed instead of only rewarding sharks if they made contact with the target, reduced the difficulty of the task and confounded experiential and social influences on learning. For this reason, it is difficult to interpret our testing phase results; however, it is clear that sharks previously paired with Ds had a 'head-start' in the learning of the task, because the only way these two groups differ is due to their earlier experience with demonstrators. Furthermore, it is also likely that a combination of low experimental quartet numbers and non-inclusion of sharks that failed to even enter the $I Z$ to initiate trials contributed to the non-significant trends in our testing phase results. The addition of these trials would also likely highlight further differences between the $\mathrm{O}$ and $\mathrm{SO}$ sharks. Future experiments, using such protocols should set learning criteria or levels for sharks to reach during individual testing. For the present study, doing this would have enabled us to assess the learning rates of sharks more clearly and at a finer scale. For example, there was a general trend for sharks to perform poorly on the first trial of each session compared with the previous day's end trial, suggesting that information was not retained well between sessions.

It has previously been hypothesised that sharks can form groups for increased foraging opportunities (Jacoby et al. 2011) and that sharks exhibiting feeding behaviour attract nearby conspecifics or heterospecifics (Myrberg et al. 1969; Bres 1993; Klimley et al. 2001). Although we did not determine specific social learning processes, our findings empirically demonstrate the sensitivity to, and likely importance of, social cues in sharks. These and other recent findings that stress the importance of social behaviour to the efficacy of shark deterrents (O'Connell et al. 2011; Robbins et al. 2011) emphasise the need for further experimentation on how changes in sharks social environment (presence of competing or informed conspecifics) might affect their foraging strategies or performance and exploitation of a novel food source. 


\section{Acknowledgments}

T.L.G. was supported by a Leverhulme Study Abroad Studentship. S.VD was supported by grants from the Marco Polo Fund and the Groningen University Fund. We thank the volunteers and staff at the Bimini Biological Field Station for their contribution. We also thank the Department of Animal Behaviour of the University of Groningen for use of their 'Observer $\mathrm{XT}$ ' behavioural analysis software. The study was financially supported by the Bimini Biological Field Station, Lacy Hoover, Earthwatch Institute, National Science Foundation (NSF-OCE 97-12793), NERC (NE/D011035/1) and Department of Education, State of Florida (FLORIDA 8749703000001). This research was carried out under a permit from Michael Braynen, Department of Fisheries of the Commonwealth of the Bahamas. We also thank anonymous reviewers for their helpful comments that improved our MS.

\section{References}

Bonfil R, Meyer M, Scholl MC et al (2005) Transoceanic migration, spatial dynamics, and population linkages of white sharks. Sci 310:100-103

Bres M (1993) The behavior of sharks. Rev Fish Biol Fish 3:133-159

Brown C, Day R (2002) The future of stock enhancements: lessons for hatchery practice from conservation biology. Fish Fish 3:79-94

Brown C, Laland KN (2001) Social learning and life skills training for hatchery reared fish. J Fish Biol 59:471-493

Brown C, Laland KN (2003) Social learning in fishes: a review. Fish Fish 4:280-288

Brown C, Laland KN (2011) Social learning in fishes. In: Brown C, Laland K, Krause J (eds) Fish cognition and behaviour. Fish and aquatic resources series. Blackwell Publishers, Cambridge. doi:10.1002/9781444342536.ch11

Bushnell P, Lutz P, Gruber SH (1989) Metabolic rate of an active tropical elasmobranch, the lemon shark Negaprion brevirostris. Exptl Biol 48(5):279-284

Clark E (1959) Instrumental conditioning of lemon sharks. Science 130:217-218

Compagno LJV (1984) FAO species catalogue. Sharks of the world: an annotated and illustrated catalogue of shark species known to date. II. Carcharhiniformes. FAO Fisheries Synopsis, Rome, Italy

DiBattista JD, Feldheim KA, Gruber SH, Hendry AP (2008) Are indirect genetic benefits associated with polyandry? Testing predictions in a natural population of lemon sharks. Mol Ecol 17:783-795

Economakis AE, Lobel PS (1998) Aggregation behaviour of the grey reef shark, Carcharhinus amblyrhychos, at Johnston Atoll, Central Pacific Ocean. Environ Biol Fish 51:129-139

Ferno" A, Huse G, Jakobsen PJ, Kristiansen TS, Nilsson J (2011) Fish behaviour, learning, aquaculture and fisheries. In: Brown C, Laland K, Krause J (eds) Fish cognition and behaviour. Fish and aquatic resources series. Blackwell Publishers, Cambridge. doi: 10.1002/9781444342536.ch16

Gruber SH (1982) Role of the lemon shark, Negaprion brevirostris (Poey), as a predator in the tropical marine environment: a multidisciplinary study. FI Sci 45:46-75

Gruber SH, Schneiderman N (1975) Classical conditioning of nictitating membrane response of lemon sharks (Negaprion brevirostris). Behav Res Meth Instru 7:430-434

Guttridge TL, Gruber SH, Gledhill KS, Croft DP, Sims DW, Krause J (2009a) Social preferences of juvenile lemon sharks, Negaprion brevirostris. Anim Behav 78:543-548

Guttridge TL, Myrberg AA, Porcher IF, Sims DW, Krause J (2009b) The role of learning in shark behaviour. Fish Fish 10:450-469

Guttridge TL, Gruber SH, DiBattista JD, Feldheim KA et al (2011) Assortative interactions and leadership in a wild population of juvenile lemon sharks. Mar Eco-Prog Ser 423:235-245 
Helfman GS, Schultz ET (1984) Social tradition of behavioural traditions in a coral reef fish. Anim Behav 32:379-384

Heyes CM (1994) Social learning in animals: categories and mechanisms. Biol Rev 69:207-231

Hoppitt W, Laland KN (2008) Social processes influencing learning in animals: a review of the evidence. Adv Study Behav 38:105-165

Jacoby DMP, Busawon DS, Sims DW (2010) Sex and social networking: the influence of male presence on social structure of female shark groups. Behav Ecol 21:808-818

Jacoby DMP, Croft DP, Sims DW (2011) Social behaviour in sharks and rays: analysis, patterns and implications for conservation. Fish Fish. doi:10.1111/j.14672979.2011.00436.x

Kessel ST (2010) An investigation into the behaviour and population dynamics of the lemon shark (Negaprion brevirostris). PhD thesis, Cardiff University, UK

Klimley AP, Nelson DR (1981) Schooling of the Scalloped Hammerhead Shark, Sphyrna-lewini, in the Gulf of California. Fish Bull 79:356-360

Klimley AP, Le Boeuf BJ, Cantara KM, Richert JE, Davis SF, Sommeran SV, Kelly JT (2001) The hunting strategy of white sharks (Carcharodon carcharias) near a seal colony. Mar Biol 138:617-636

Krause J, Ruxton GD (2002) Living in groups. Oxford series in ecology and evolution. Oxford University Press, Oxford

Laland KN, Atton N, Webster MM (2011) From fish to fashion: experimental and theoretical insights into the evolution of culture. Phil Trans R Soc B 366:958-968. doi:10.1098/rstb.2010.0328

Leadbeater E, Chittka L (2007) The dynamics of social learning in an insect model, the bumblebee (Bombus terrestris). Behav Ecol Sociobiol 61:1789-1796

Mineka S, Cook M (1988) Social learning and the acquisition of snake fear in monkeys. In: Zentall TR, Galef BF (eds) Social learning: psychological and biological perspectives. Lawrence Erlbaum Associates, New Jersey, pp 51-74

Myrberg AA, Gruber SH (1974) The behavior of the bonnethead shark, Sphyrna tiburo. Copeia 1974:358374

Myrberg AA, Banner A, Richard JD (1969) Shark attraction using a video-acoustic system. Mar Biol 2:264-276

Nelson DR, Johnson RH (1980) Behavior of reef sharks of Rangiroa, French Polynesia. Nat Geo Soc Res Rep 12:479-499

Nixon A, Gruber SH (1988) Diel metabolic and activity patterns of the lemon shark (Negaprion brevirostris). J Exptl Zool 248(1):1-6

Noldus IT (2007) The Observer XT. Information Technology O'Connell CP, Abel DC, Stroud EM, Rice PH (2011) Analysis of permanent magnets as elasmobranch by catch reduction devices in hook-andline and longline trials. Fish Bull 109:394-401

Powter DM, Gladstone W (2009) Habitat-mediated use of space by juvenile and mating adult port jackson sharks, Heterodontus portusjacksoni, in Eastern Australia. Pac Sci 63:1-14

Robbins WD, Peddemors VM, Kennelly SJ (2011) Assessment of permanent magnets and electropositive metals to reduce the linebased capture of Galapagos sharks, Carcharhinus galapagensis. Fish Res 109:100-106

Ryer CH, Olla BL (1992) Social mechanisms facilitating exploitation of spatially variable ephemeral food patches in a pelagic marine fish. Anim Behav 44:69-74

Speed CW, Field IC, Meekan MG, Bradshaw CJA (2010) Complexities of coastal shark movements and their implications for management. Mar Ecol Prog Ser 408:275-293

Springer S (1967) Social organization of shark populations. In: Gilbert PW, Mathewson RF, Rall DP (eds) Sharks, skates and rays. John Hopkins Press, Baltimore, pp 149-174

Vilhunen S (2006) Repeated anti-predator conditioning: a pathway to habituation or to better avoidance? J Fish Biol 68:25-43 
Wetherbee BM, Gruber SH, Rosa RS (2007) Movement patterns of juvenile lemon sharks Negaprion brevirostris within Atol das Rocas, Brazil: a nursery characterized by tidal extremes. Mar Ecol Prog Ser 343:283-293 